\title{
Biocatalytic Hybrid Nanoparticles for Degassing and Stabilization of Single Electron Transfer-Living Radical Polymerization in Pickering emulsions
}

\author{
Adrian Moreno Guerra \\ Stockholm University \\ Mika Sipponen ( $\sim$ mika.sipponen@mmk.su.se) \\ Stockholm University https://orcid.org/0000-0001-7747-9310
}

\section{Article}

Keywords: Pickering emulsions, SET-LRP, controlled radical polymerization, lignin, hybrid nanoparticles

Posted Date: August 4th, 2020

DOI: https://doi.org/10.21203/rs.3.rs-48599/v1

License: (c) (1) This work is licensed under a Creative Commons Attribution 4.0 International License.

Read Full License

Version of Record: A version of this preprint was published at Nature Communications on November 5th, 2020. See the published version at https://doi.org/10.1038/s41467-020-19407-3. 


\section{Abstract}

Synthetic polymers are indispensable in many different applications, but there is a growing need for green processes and natural surfactants for emulsion polymerization. The use of solid particles to stabilize Pickering emulsions is a particularly attractive avenue, but oxygen sensitivity has remained a formidable challenge in controlled polymerization reactions. Here we show that lignin nanoparticles (LNPs) coated with chitosan and glucose oxidase (GOx) enable efficient stabilization of Pickering emulsion and in situ enzymatic degassing of single electron transfer-living radical polymerization (SETLRP) without extraneous hydrogen peroxide scavengers. The resulting latex dispersions can be purified by aqueous extraction or used to obtain polymer nanocomposites containing uniformly dispersed LNPs. The polymers exhibit high chain-end fidelity that allows for production of a series of well-defined block copolymers as a viable route to more complex architectures.

\section{Introduction}

Reversible deactivation radical polymerization (RDRP) techniques have emerged as one of the main tools for the efficient and precise synthesis of macromolecules with sophisticated and well-defined architectures for a wide range of macromolecular engineering applications. ${ }^{[1-5]}$ Atom transfer radical polymerization (ATRP), reversible addition-fragmentation (RAFT) polymerization and single electron transfer- living radical polymerization (SET-LRP) offer high versatility to produce polymers, and the ability to minimize the termination events, while favoring equal propagation of polymeric chains during the polymerization process. ${ }^{[6-8]}$

One of the main challenges of RDRP techniques arises from the presence of oxygen that is a well-known and undesired radical scavenger, ${ }^{[9]}$ which forces the application of degassing protocols or the introduction of reducing agents (e.g. hydrazine), ${ }^{[10][11]}$ thus hampering the scale-up and transition from academia to industry of these controlled polymerization processes. To overcome this issue, notable works from the groups of Steven ${ }^{[12-14]}$ and Matyjaszweski, ${ }^{[15-17]}$ among others ${ }^{[18-21]}$ reported the use of oxidoreductase enzymes to deplete oxygen, thus giving rise to enzyme-degassed RDRP techniques. Glucose oxidase (GOx, EC 1.1.3.4) is by far the most common enzyme applied due its commercial availability and high efficiency to consume oxygen from the reaction mixture, allowing efficient polymerization processes in open-air systems. ${ }^{[12][13]}$ So far, most of these systems have been applied in RAFT and ATRP of water-soluble monomers in aqueous media to retain the enzyme activity. ${ }^{[16-20]}$ The polymerization of hydrophobic monomers remains scarcely explored, and is hitherto limited to ATRPmediated miniemulsion processes using soluble ionic surfactants such as dodecyl sulfate (SDS), ${ }^{[17]}$ which could disrupt the activity of the enzyme at longer reactions times. ${ }^{[22]}$ Indeed, some of these previous reported systems require the addition of sacrificial substrates (e.g. sodium pyruvate) to act as oxidant scavengers to consume the hydrogen peroxide generated during the GOx-catalyzed degassing step and thereby avoid giving rise to detrimental Fenton-like redox processes. ${ }^{[15]}$ 
Taking into account the growing interest towards polymerization in dispersed media in both academia and industry, one of the current challenges is to develop surfactant/emulsifier systems that stabilize emulsions of hydrophobic vinyl monomers for RDRP, while simultaneously enabling enzymatic deoxygenation of water and scavenging of the hydrogen peroxide formed during the degassing step. Pickering emulsion polymerization stabilized by organic or inorganic particles has gained a great attention over conventional emulsion polymerization process due to a more stable emulsification behavior, and the ability to produce composites with improved properties. ${ }^{[23][24]}$ These systems have been scarcely applied in RDRP techniques such as ATRP or RAFT to generate hybrid latexes with a reasonable extent of control. ${ }^{[25][26]}$ In addition, the literature is lacking reports on the development of SET-LRP mediated Pickering emulsion processes in general and enzyme-degassed RDRP-mediated Pickering emulsion processes in particular.

Here we report a multifunctional Pickering emulsion stabilizer that enables efficient SET-LRP of waterimmiscible vinyl monomers without extra degassing steps and at a broad temperature range. Lignin nanoparticles (LNPS) coated with cationic polymers act as the carrier for GOx because of their recently reported beneficial properties in stabilization of Pickering emulsions ${ }^{[27][28]}$ and enzyme immobilization. ${ }^{[29]}$ [30] In addition to investigating the ability of the system to scavenge the in situ generated hydrogen peroxide and produce well-defined polymers, we also explore the obtained latex dispersions for the preparation of lignin-based composites. We demonstrate that a simple melt processing of the latex dispersions gives composites with uniform dispersion of antioxidant and UV-protective nanoparticles in the polymeric matrixes, resolving in this way one of the main challenges in the preparation of composites with bio-based particulate fillers.

\section{Results}

Fabrication of biocatalytic hybrid particles. Our approach to fabricate multifunctional Pickering emulsion polymerization stabilizers started with production of biocatalyst loaded LNPs (GOx-chi-LNPs) via twostep adsorption immobilization strategy (Fig. 1).

First, LNPs ( $\zeta$-potential - $40 \mathrm{mV}$ ) were produced by solvent-exchange methodology from previously characterized pine kraft lignin (Table S1). ${ }^{[29]}$ The resulting LNPs (diameter $97 \mathrm{~nm}$ ) were used to adsorb chitosan (10 wt\% relative to LNPs) as a cationic polyelectrolyte. As previously reported, ${ }^{[28]}$ we obtained colloidally stable cationic chitosan-coated LNPs (chi-LNPs) ( $\zeta$-potential $+32 \mathrm{mV}$ and diameter $190 \mathrm{~nm}$ ) that were here used to adsorb GOx (10 mg g-1 chi-LNPs) to yield the enzyme-immobilized LNPs (GOx-chiLNPs) (Figure S1 and S2). The increase in size and surface charge as determined by dynamic light scattering (DLS) analysis of the resulting particles after the two-step adsorption procedure $(215 \mathrm{~nm}$ and $+41 \mathrm{mV}$ ) verified an effective electrostatic interaction between GOx and chi-LNPs (Fig. 2a-b and table $\mathrm{s} 2)$.

Scanning electron microscopy (SEM) images confirmed the formation of spherical and uniformly shaped biocatalyst-loaded particles (GOx-chi-LNPs) that exhibited a high tendency to agglomerate upon drying 
(Fig. 2c). A protein mass balance based on Bradford protein assay (BSA standards), showed that $88 \%$ of GOx adsorbed on chi-CLPs, while the remaining $12 \%$ was present in solution or adsorbed on the smallest particles that did not sediment during the centrifugation step used in the sample preparation for the protein assay.

Since polymerization reactions are carried out in a broad range of temperatures, we first studied thermal stability of the immobilized GOx in comparison to the free enzyme. It turned out that the immobilized GOx remained active until $80^{\circ} \mathrm{C}$, with a temperature optimum at $65^{\circ} \mathrm{C}$ compared to about $50{ }^{\circ} \mathrm{C}$ of the free enzyme (Fig. 2d). One possible explanation for this increased stability is the ability of the chi-LNPs to scavenge hydrogen peroxide produced by GOx (Fig. 1, and vide infra for more details). Comparison before and after the immobilization step of enzyme activity also confirmed a high retention of activity, which could be associated to a low kinetic constraint from the embedment of the enzyme on the chitosan hydrogel layer over the LNPs (Figure S3 and Table S3).

\section{Enzyme-degassed SET-LRP-mediated Pickering emulsion using GOx-chi-LNPs as emulsifiers. With the} biocatalyst-loaded particles available as a colloidally stable dispersion, we assessed whether or not the biocatalytic emulsifier system is suitable for conducting SET-LRP reactions. All the polymerization were conducted in aqueous buffer solutions $(\mathrm{pH}=6)$ at $50^{\circ} \mathrm{C}$ in Pickering emulsions produced by mixing the monomer, ligand and initiator (oil phase) with an aqueous dispersion containing GOx-chi-LNPs (20 g per $\mathrm{L}$ of monomer) and glucose $(0.1 \mathrm{M})$ by ultrasonication (See methods section and Table $\mathrm{S} 4$ for more details of polymerization conditions). The emulsion droplets were efficiently covered by GOx-chi-LNPs (Figure S4 and Table S5) regardless of the monomer employed, and polymerization was initiated by adding a small volume of aqueous $\mathrm{Cu}(0)$ powder dispersion into the Pickering emulsion. Nanosized $\mathrm{Cu}(0)$ particles $(40-60 \mathrm{~nm}$ ) were used to ensure a high active surface area and provide a fast initiation step, crucial to the domain propagation step. ${ }^{[31]}$ The polymerization reactions were allowed to run in either open or closed vials.

The feasibility of GOx-chi-LNPs to provide an oxygen tolerant and controlled polymerization process was evaluated by the polymerization of butyl methacrylate (BMA) as a model monomer in closed vials to avoid the loss of volatile BMA from the reaction mixture (Fig. 3a). A kinetic polymerization study revealed a pseudo-first-order polymerization kinetics and an excellent linearity between $\ln \left(\left[\mathrm{M}_{0}\right] /[\mathrm{M}]\right)$ vs time (Fig. 3b, circles), indicating an equal and constant propagation of the growing polymeric chains, the principal feature of a living radical polymerization process. In addition, any appreciable induction period could not be observed, which confirms an efficient degassing process catalyzed from GOx-chi-LNPs. As a visual observation it was noted that the appearance of the emulsion became more opaque as a result of the polymerization (Fig. 3c-d). The high tolerance to air atmosphere was additionally assed by temporally opening and closing the reaction vial in 20 minutes intervals during 4 hours, which essentially confirmed that exposure to air had no effect on the polymerization rate or extent of control even at the most oxygenexposed periods (open vial times) (Figure S5). For comparison, chi-LNPs without GOx were used by applying nitrogen gas purging as a degassing methodology (Fig. 3b, triangles) and without any degassing protocol (Fig. 3b, squares). It can be very clearly observed that the system of chi-LNPs without 
degassing measures showed an induction period of more than $3 \mathrm{~h}$ before the polymerization began to proceed, which confirms previous findings on the inhibition of the reaction by dissolved oxygen. ${ }^{[10]}$ In the case of chi-LNPs, applying $\mathrm{N}_{2}$ bubbling for degassing, a similar kinetic profile than that observed for GOxchi-LNPs could be obtained, albeit at a slightly slower polymerization rate, which could be associated to the inevitable oxygen contamination resulting from the sampling process despite applying concurrent purging with $\mathrm{N}_{2}$.

These results not only validate our original hypothesis and confirm GOx-chi-LNPs as efficient degassing stabilizers for Pickering emulsions polymerizations, but also demonstrate that GOx-chi-LNPs can provide even a faster polymerization rate than chi-LNPs after applying nitrogen purging, which could be of potential interest for industrial applications. Moreover, molecular weight analysis at different polymerization times by GPC revealed a linear increase of molar mass with an excellent agreement with the theoretical molecular weight values, leading to well defined polymers $\left(M_{w} / M_{n}=1.30-1.15\right)$ during the whole polymerization process regardless of the type of LNPs (chi-LNPs or GOx-chi-LNPs) used as emulsifiers (Fig. 3e and S6). Therefore, these results validated the potential of lignin nanoparticles as functional emulsifiers for SET-LRP.

Self-scavenger ability of GOx-chi-LNPs towards hydrogen peroxide. Inactivation by hydrogen peroxide of enzymes in general and GOx in particular is a problem that has been alleviated by additives such as the electron acceptor system benzoquinone-hydroquinone. ${ }^{[32]}$ Moreover, as already mentioned, $\mathrm{H}_{2} \mathrm{O}_{2}$ may initiate Fenton-like oxidation processes and promote the generation of new chains, leading to uncontrolled growing of the polymeric chains and thus affecting the final molecular weight distribution. ${ }^{[15]}$ It is thus important to note that our system was able to generate well-defined polymers without the need of any extraneous reducing agents (e.g. sodium pyruvate) to eliminate the $\mathrm{H}_{2} \mathrm{O}_{2}$ formed during the degassing step. This fact could be attributed to the inherent nature of both chitosan and lignin to consume $\mathrm{H}_{2} \mathrm{O}_{2}$ in situ. ${ }^{[33][34]}$ To test this hypothesis, we evaluated the ability of GOx-chi-LNPs to consume $\mathrm{H}_{2} \mathrm{O}_{2}$. First, $\mathrm{H}_{2} \mathrm{O}_{2}$ was dissolved in an aqueous solution $(\mathrm{pH}=6)$ and mixed with horseradish peroxidase (HRP, EC 1.11.1.7) in opened vials with and without LNPs or GOx-chi-LNPs, and then o-dianisidine was added as a substrate for HRP (Figure S7a). In the absence of LNPs or GOx-chi-LNPs, a significant increase in absorption at $500 \mathrm{~nm}$ was observed by UV-vis spectroscopy due to the formation of the oxidized dimer of $o$-dianisidine $\left(\varepsilon_{[500 \mathrm{~nm}]}=7.5 \mathrm{mM}\right.$ in water) catalyzed by HRP in the presence of $\mathrm{H}_{2} \mathrm{O}_{2}$ (Figure S7b). In contrast, when the reaction was performed in the presence of LNPs, a noteworthy decrease in the UV absorption of oxidized $o$-dianisidine indicated an effective consumption of $\mathrm{H}_{2} \mathrm{O}_{2}$ by LNPs (Figure S7c and S7e). Additionally, it was interesting to observe that when GOx-chi-LNPs were added to the reaction mixture, only trace levels of the oxidized product could be determined, suggesting an excellent combined effect of lignin and chitosan to scavenge the $\mathrm{H}_{2} \mathrm{O}_{2}$ present in the system. (Figure S7d). Additionally, to mimic the polymerization conditions, the same amount of BMA, glucose and GOXchi-LNPs were dissolved in an aqueous solution $(\mathrm{pH}=6)$ and exposed to air. Then, $o$-dianisidine and HRP solution were added, and the analysis of the resulting solution confirmed that the mere presence of GOx- 
chi-LNPs suppressed the $\mathrm{H}_{2} \mathrm{O}_{2}$ formed in the reaction media (Figure S8). These results confirm the scavenger ability of our system towards $\mathrm{H}_{2} \mathrm{O}_{2}$, which is beneficial not only for the polymerization process but also to protect the enzyme against the oxidative denaturation, ${ }^{[35]}$ which could also explain the high retention of enzyme activity that the immobilized GOx exhibited at high temperatures (Fig. 2d).

Applicability to other monomers and synthesis of well-defined block copolymers. Having demonstrated the robust nature of GOx-chi-LNPs to act as functional surfactant to obtain well-defined hydrophobic polymers from oxygen-tolerant SET-LRP, we explored versatility of the system by targeting the polymerization of different vinyl monomer of relevant industrial interest. For this propose, we conducted polymerizations under identical degree of polymerization $(D P=200)$ to those applied for BMA for two additional hydrophobic monomers, namely methyl acrylate (MA) and styrene (S). In both cases, near to quantitative monomer conversion (90\%) was achieved, and molecular weight analysis of the resulting polymers revealed symmetrical monomodal peaks $\left(M_{w} / M_{n}=1.16\right.$ for $M A$ and 1.26 for $\left.S\right)$ with an excellent agreement between the experimental and theoretical molecular weights values (Fig. 4a), proving the versatility of this system to polymerize, in a controlled manner, different families of hydrophobic vinyl monomers in aqueous phase. We also decided to push the limits of our system by targeting different degrees of polymerization $\left(D P_{n}\right)$, ranging from 50 to 500 for BMA. The results are summarized in Table S6. In all cases, well-defined polymers with narrow dispersities $\left(M_{w} / M_{n}=1.29-1.16\right)$ and experimental molecular weight values close to the theoretical ones could be obtained, indicating a high versatility of the system to deliver well defined polymers in a wide range of molecular weights (Fig. 4b and Figure S9).

The high chain-end fidelity of the obtained polymers, a critical parameter to synthetize more complex architectures (e.g. block and multiblock copolymers) ${ }^{[36-38]}$ was also assed for our system. Low molecular weight PMA ( $\mathrm{M} / \mathrm{I}=50)$ was synthetized, isolated and analyzed by ${ }^{1} \mathrm{H}$ NMR spectroscopy, which revealed virtually complete chain-end functionality (96\%) based on the retention of the bromine chain-ends (Figure S10). Indeed, chain extension experiments by stopping the reaction, purification of the macromonomer through precipitation and re-dispersion to continue the polymerization of a second block also confirmed a nearly perfect chain-end functionality regardless of the monomer employed (Fig. 4c and S11). The successful formation of quasi real AB block copolymers was confirmed by a clear shift in the GPC curves towards high molecular weight values without noticeable shoulders or tailing (Fig. 4c and S11), indicating the absence of unreactive polymeric chain produced in recombination processes of growing polymeric chains (e.g. bimolecular termination processes). Overall, these results not only validate GOx-chi-LNPs as an efficient stabilizers for more sophisticated controlled polymerization techniques under the presence of oxygen, but also revealed their high versatility to stabilize and enable SET-LRP of different vinyl monomers leading to well-defined polymers as building units for more complex and sophisticated architectures.

Analysis of latex dispersion and production of lignin-based composites. Finally, the quality of the latex dispersions obtained after the SET-LRP process was also evaluated by SEM and optical light microscopy. Regardless of the monomer employed, the latex dispersions were in all cases composed of uniform 
spherical polymeric microbeads (Fig. 5 and Figure S12), with a similar size to that of the initial monomer droplets (Compare Table S5 and S7). As a representative example, BMA latex dispersion was selected for a more detailed analysis (Fig. 5). Lignin-coated PBMA microparticles could be obtained by isolation of the particles by a simple centrifugation/re-dispersion step. Based on the microscopic images, it can be clearly observed that the PBMA microspheres contained a thin amorphous layer (Fig. 5b and c), which demonstrate the high emulsifier capacity of GOx-chi-LNPs system. Such a uniform distribution of the nanoparticles in the polymeric matrix inspired us to envision that the polymerization system could also provide an effective and direct method to prepare lignin-based composites with homogeneously dispersed lignin particles in the polymer matrix. With this objective in mind, we evaluated the preparation of composites by simple melting at $160^{\circ} \mathrm{C}$ of the PBMA latex dispersion stabilized with a higher content of GOx-chi-LNPs ( 6 wt \% relative to BMA) (Figure S13a). SEM analysis of the surface and cross sections of PBMA-GOx-chi-LNPs film composites (Figure S13b-c) confirmed the uniform distribution of GOx-chiCLPs without agglomeration within the PBMA matrix, which indicates an unprecedented and effective interaction of the particles even within such hydrophobic polymeric matrixes. These results make it possible to form lignin-based polymer composites in a simple way, avoiding chemical functionalization of lignin to improve the dispersability within the polymeric matrix. Last but not least, it is also important to note that highly purified PBMA microparticles without the presence of lignin could also be obtained by simple aqueous basic extraction of the latex dispersions under alkaline conditions (Fig. 5d-f).

\section{Discussion}

We have developed a multifunctional particulate emulsifier based on enzyme-immobilized lignin nanoparticles (GOx-chi-LNPs), and demonstrated their application in oxygen-tolerant SET-LRP in Pickering emulsions. This constitutes the first report on enzyme-degassed SET-LRP reactions, and enzymedegassed RDRP-mediated Pickering emulsion processes in particular. The rational design of our GOx-chiLNPs was not only limited to enable the synthesis of hydrophobic polymers in a controlled manner under air atmosphere, but also to enhance the enzyme activity in a wide range of temperatures, together with a self-scavenger ability towards $\mathrm{H}_{2} \mathrm{O}_{2}$ during the polymerizations without adding external additives. Additionally, the potential of this approach has been highlighted by the efficient preparation of ligninbased composites, with an expected favorable carbon footprint by simple melt processing. Finally, we also hold a view that this system can be easily transferred to other polymerization techniques such as ATRP, RAFT or FRP and thus holds potential for opening new avenues in the preparation of precision polymers and lignin-polymer composites under green conditions.

\section{Methods}

Preparation of colloidal lignin particles (LNPs, chi-LNPs and GOx-chi-LNPs). LNPs used in this work were produced following the same procedure described earlier with a few modifications. ${ }^{[28]}$ Briefly, kraft lignin was dissolved in acetone/water mixture (mass ratio 3:1), insoluble impurities were removed by filtration, and LNPs produced by rapid pouring of deionized water to lignin solution followed by rotary evaporation 
of acetone. The final aqueous dispersion of LNPs (0.4 wt\%) was obtained with a lignin mass yield of $89 \%$. The chitosan coated LNPs (chi-LNPs) were prepared by adding the dispersion of LNPs under vigorous stirring into a $0.1 \mathrm{wt} \%$ chitosan solution. The ratio of chitosan to LNPs was $100 \mathrm{mg} \mathrm{g}^{-1}$. GOxchi-LNPs were produced by the addition of a glucose oxidase (GOx) solution (sodium acetate buffer, $0.1 \mathrm{M} ; \mathrm{pH}$ 5) to chi-LNPs dispersion under orbital shaking at room temperature for $2 \mathrm{~h}$. GOx-chi-LNPs were recovered by three centrifugation/re-dispersion cycles (3000 rpm, $10 \mathrm{~min}$ ), replacing each decanted supernatant with sodium acetate buffer $(0.1 \mathrm{M} ; \mathrm{pH} 5)$. The ratio of GOx to chi-CLPs was $10 \mathrm{mg} \mathrm{g}^{-1}$.

Preparation of GOx-chi-LNPs stabilized BMA-in-water Pickering emulsions. All the emulsions were prepared by gradually adding BMA monomer to a water dispersion of GOx-chi-LNPs. The final fraction of oil/water was fixed at $20 / 80 \mathrm{v} / \mathrm{v}$ and the total volume of the emulsion was $10 \mathrm{~mL}$. The final concentration of GOx-chi-CLPs was fixed to $20 \mathrm{~g}$ of GOx-chi-CLPs per L (2.25 wt\%) of BMA. The emulsification was performed by sonication for $120 \mathrm{~s}$ with a BioBlock Vibra-Cell equipped with an ultrasonic tip with cooling in an ice bath ( $10 \mathrm{~s}$ on and $5 \mathrm{~s}$ off at $40 \%$ of amplitude power).

Enzyme-degassed SET-LRP-mediated Pickering emulsion process. This procedure is representative for all the polymerization conducted herein. The enzyme-degassed Pickering emulsion SET-LRP of BMA using GOX-chi-LNPs as emulsifier was carried out under the following conditions: $[\mathrm{BMA}]_{0} /[\mathrm{MBPA}]_{0} /\left[\mathrm{Me}_{6^{-}}\right.$ TREN $]_{0} /[\mathrm{Cu}(0)]_{0}=200 / 1 / 0.2 / 0.3$. [GOx-chi-LNPs] $=2.25 \mathrm{wt} \%$ relative to BMA, is described as a representative procedure. A stock solutions of BMA ( $2 \mathrm{~mL}, 0.012 \mathrm{~mol}), \operatorname{MBPA}(11 \mu \mathrm{L}, 0.062 \mathrm{mmol})$ and $\mathrm{Me}_{6}$-TREN (3.36 $\mu \mathrm{L}, 0.012 \mathrm{mmol}$ ) were prepared. GOx-chi-LNPs (40 mg, $2.25 \mathrm{wt} \%$ to BMA) were dispersed in water $(7.5 \mathrm{~mL})$ containing glucose $(200 \mathrm{mg}, 0.14 \mathrm{M})$. Then, BMA-in-water Pickering emulsion were prepared by ultrasonication of organic phase (oil phase) in water phase as described above. The Pickering emulsion was transferred to a vial and placed in a thermostatic oil-bath at $50{ }^{\circ} \mathrm{C}$. The introduction of an aliquot of $\mathrm{Cu}(0)$ powder dispersed in water $(1.20 \mathrm{mg}, 0.5 \mathrm{~mL})$ started the SET-LRP process $(t=0)$. In general, the reactions were allowed to proceed during $12 \mathrm{~h}$, and in the case of kinetic experiments, samples were withdrawn periodically to follow the monomer conversion by gravimetric analysis. The resulting lignin-polymeric microparticles were purified by three centrifugation/re-dispersion cycles, replacing each decanted supernatant with aqueous basic solution $(\mathrm{NaOH}, 0.1 \mathrm{M})$, followed by drying overnight of the purified polymer samples at $45^{\circ} \mathrm{C}$ for $12 \mathrm{~h}$ prior to the GPC analysis. To purify the polymeric particles without removing lignin from microparticle surface, deionized water was used instead of basic washing solution.

\section{ASSOCIATED CONTENT}

\section{Supporting Information}

The Supporting Information is available free of charge: Materials, additional experimental procedures, characterization techniques and additional data: SEM images for the particles, enzymatic studies, additional GPC traces for the different experiments, additional SEM and optical microscope images from 
the microparticles and latex dispersions, chain end analysis via ${ }^{1} \mathrm{H} N \mathrm{NR}$, and analysis of composites by SEM and digital images.

\section{Declarations}

\section{AUTHOR CONTRIBUTIONS}

A.M. and M.H.S conceived the idea and designed the experiments. A.M. performed the experiments and analyzed the data with inputs from M.H.S. A.M and M.H.S co-wrote the manuscript.

\section{ACKNOWLEDGMENT}

The authors acknowledge the Department of Materials and Environmental Chemistry (MMK) for financing this work through the start-up grant awarded to MHS. The authors also acknowledge Dr. Kjell Jansson for helpful discussions, and Prof. Lennart Bergström and Dr. Claudia Möckel for giving access to ultrasonication and GPC instrumentation, respectively.

\section{References}

1. Anastasaki, A. et al. Cu(0)-Mediated Living Radical Polymerization: A Versatile Tool for Materials Synthesis. Chem. Rev. 116, 835-877 (2016).

2. Matyjaszewski, K. Advanced Materials by Atom Transfer Radical Polymerization. Adv. Mater. 30, 122 (2018).

3. Baker, S. L. et al. Atom Transfer Radical Polymerization for Biorelated Hybrid Materials. Biomacromolecules 20, 4272-4298 (2019).

4. Lligadas, G., Grama, S. \& Percec, V. Recent Developments in the Synthesis of Biomacromolecules and their Conjugates by Single Electron Transfer-Living Radical Polymerization. Biomacromolecules 18, 1039-1063 (2017).

5. Niu, J. et al. Engineering live cell surfaces with functional polymers via cytocompatible controlled radical polymerization. Nat. Chem. 9, 537-545 (2017).

6. Lligadas, G., Grama, S. \& Percec, V. Single-Electron Transfer Living Radical Polymerization Platform to Practice, Develop, and Invent. Biomacromolecules 18, 2981-3008 (2017).

7. Perrier, S. 50th Anniversary Perspective: RAFT Polymerization - A User Guide. Macromolecules 50, 7433-7447 (2017).

8. Matyjaszewski, K. Atom Transfer Radical Polymerization (ATRP): Current status and future perspectives. Macromolecules 45, 4015-4039 (2012).

9. Yeow, J., Chapman, R., Gormley, A. J. \& Boyer, C. Up in the air: Oxygen tolerance in controlled/living radical polymerisation. Chem. Soc. Rev. 47, 4357-4387 (2018). 
10. Sven, F., Rosen, B. M. \& Virgil, P. SET-LRP of acrylates in Air. J. Polym. Sci. Part A Polym. Chem. 48, 1190-1196 (2010).

11. Moreno, A. et al. SET-LRP of Bio- and Petroleum-Sourced Methacrylates in Aqueous Alcoholic Mixtures. Biomacromolecules 20, 1816-1827 (2019).

12. Mohammed, A. A. et al. Open vessel free radical photopolymerization of double network gels for biomaterial applications using glucose oxidase. J. Mater. Chem. B 7, 4030-4039 (2019).

13. Chapman, R., Gormley, A. J., Stenzel, M. H. \& Stevens, M. M. Combinatorial Low-Volume Synthesis of Well-Defined Polymers by Enzyme Degassing. Angew. Chem. Int. Ed. 55, 4500-4503 (2016).

14. Chapman, R., Gormley, A. J., Herpoldt, K. L. \& Stevens, M. M. Highly controlled open vessel RAFT polymerizations by enzyme degassing. Macromolecules 47, 8541-8547 (2014).

15. Enciso, A. E., Fu, L., Russell, A. J. \& Matyjaszewski, K. A Breathing Atom-Transfer Radical Polymerization: Fully Oxygen-Tolerant Polymerization Inspired by Aerobic Respiration of Cells. Angew. Chem. Int. Ed. 57, 933-936 (2018).

16. Navarro, L. A., Enciso, A. E., Matyjaszewski, K. \& Zauscher, S. Enzymatically Degassed SurfaceInitiated Atom Transfer Radical Polymerization with Real-Time Monitoring. J. Am. Chem. Soc. 141, 3100-3109 (2019).

17. Wang, Y., Fu, L. \& Matyjaszewski, K. Enzyme-Deoxygenated Low Parts per Million Atom Transfer Radical Polymerization in Miniemulsion and Ab Initio Emulsion. ACS Macro Lett. 7, 1317-1321 (2018).

18. Oytun, F., Kahveci, M. U. \& Yagci, Y. Sugar overcomes oxygen inhibition in photoinitiated free radical polymerization. J. Polym. Sci. Part A Polym. Chem. 51, 1685-1689 (2013).

19. Wang, M. et al. Enzyme Degassing for Oxygen-Sensitive Reactions in Open Vessels of an Automated Parallel Synthesizer: RAFT Polymerizations. ACS Comb. Sci. 21, 643-649 (2019).

20. Liu, Z., Lv, Y. \& An, Z. Enzymatic Cascade Catalysis for the Synthesis of Multiblock and UltrahighMolecular-Weight Polymers with Oxygen Tolerance. Angew. Chem. Int. Ed. 56, 13852-13856 (2017).

21. Schneiderman, D. K. et al. Open-to-Air RAFT Polymerization in Complex Solvents: From Whisky to Fermentation Broth. ACS Macro Lett. 7, 406-411 (2018).

22. Jones, M. N., Manley, P. \& Wilkinson, A. The dissociation of glucose oxidase by sodium n-dodecyl sulphate. Biochem. J. 203, 285-291 (1982).

23. Werner, A., Sèbe, G. \& Héroguez, V. A new strategy to elaborate polymer composites: Via Pickering emulsion polymerization of a wide range of monomers. Polym. Chem. 9, 5043-5050 (2018).

24. Colver, P. J., Colard, C. A. L. \& Bon, S. A. F. Multilayered nanocomposite polymer colloids using emulsion polymerization stabilized by solid particles. J. Am. Chem. Soc. 130, 16850-16851 (2008).

25. Werner, A., Schmitt, V., Sèbe, G. \& Héroguez, V. Convenient Synthesis of Hybrid Polymer Materials by AGET-ATRP Polymerization of Pickering Emulsions Stabilized by Cellulose Nanocrystals Grafted with Reactive Moieties. Biomacromolecules 20, 490-501 (2019). 
26. Zhou, J., Yao, H. \& Ma, J. Recent advances in RAFT-mediated surfactant-free emulsion polymerization. Polym. Chem. 9, 2532-2561 (2018).

27. Sipponen, M. H., Smyth, M., Leskinen, T., Johansson, L. S. \& Österberg, M. All-lignin approach to prepare cationic colloidal lignin particles: Stabilization of durable Pickering emulsions. Green Chem. 19, 5831-5840 (2017).

28. Zou, T., Sipponen, M. H. \& Österberg, M. Natural shape-retaining microcapsules with shells made of chitosan-coated colloidal lignin particles. Front. Chem. 7, 370 (2019).

29. Sipponen, M. H. et al. Spatially confined lignin nanospheres for biocatalytic ester synthesis in aqueous media. Nat. Commun. 9, 2300 (2018).

30. Capecchi, E. et al. Enzyme-Lignin Nanocapsules Are Sustainable Catalysts and Vehicles for the Preparation of Unique Polyvalent Bioinks. Biomacromolecules 20, 1975-1988 (2019).

31. Lligadas, G., Rosen, B. M., Bell, C. A., Monteiro, M. J. \& Percec, V. Effect of Cu(0) particle size on the kinetics of SET-LRP in DMSO and Cu-mediated radical polymerization in MeCN at $25^{\circ} \mathrm{C}$. Macromolecules 41, 8365-8371 (2008).

32. Bourdillon, C., Hervagault, C. \& Thomas, D. Increase in operational stability of immobilized glucose oxidase by the use of an artifical cosubstrate. Biotechnol. Bioeng. 27, 1619-1622 (1985).

33. Chang, K. L. B., Tai, M. C. \& Cheng, F. H. Kinetics and products of the degradation of chitosan by hydrogen peroxide. J. Agric. Food Chem. 49, 4845-4851 (2001).

34. He, W., Gao, W. \& Fatehi, P. Oxidation of Kraft Lignin with Hydrogen Peroxide and its Application as a Dispersant for Kaolin Suspensions. ACS Sustain. Chem. Eng. 5, 10597-10605 (2017).

35. Alberti, B. N. \& Klibanov, A. M. Preparative production of hydroquinone from benzoquinone catalysed by immobilized d-glucose oxidase. Enzyme Microb. Technol. 4, 47-49 (1982).

36. Anastasaki, A. et al. One-Pot Synthesis of ABCDE Multiblock Copolymers with Hydrophobic, Hydrophilic, and Semi-Fluorinated Segments. Angew. Chem. Int. Ed. 56, 14483-14487 (2017).

37. Moreno, A. et al. Macromonomers, telechelics and more complex architectures of PMA by a combination of biphasic SET-LRP and biphasic esterification. Polym. Chem. 9, 1885-1899 (2018).

38. Moreno, A., Liu, T., Galià, M., Lligadas, G. \& Percec, V. Acrylate-macromonomers and telechelics of PBA by merging biphasic SET-LRP of BA, chain extension with MA and biphasic esterification. Polym. Chem. 9, 1961-1971 (2018).

\section{Figures}




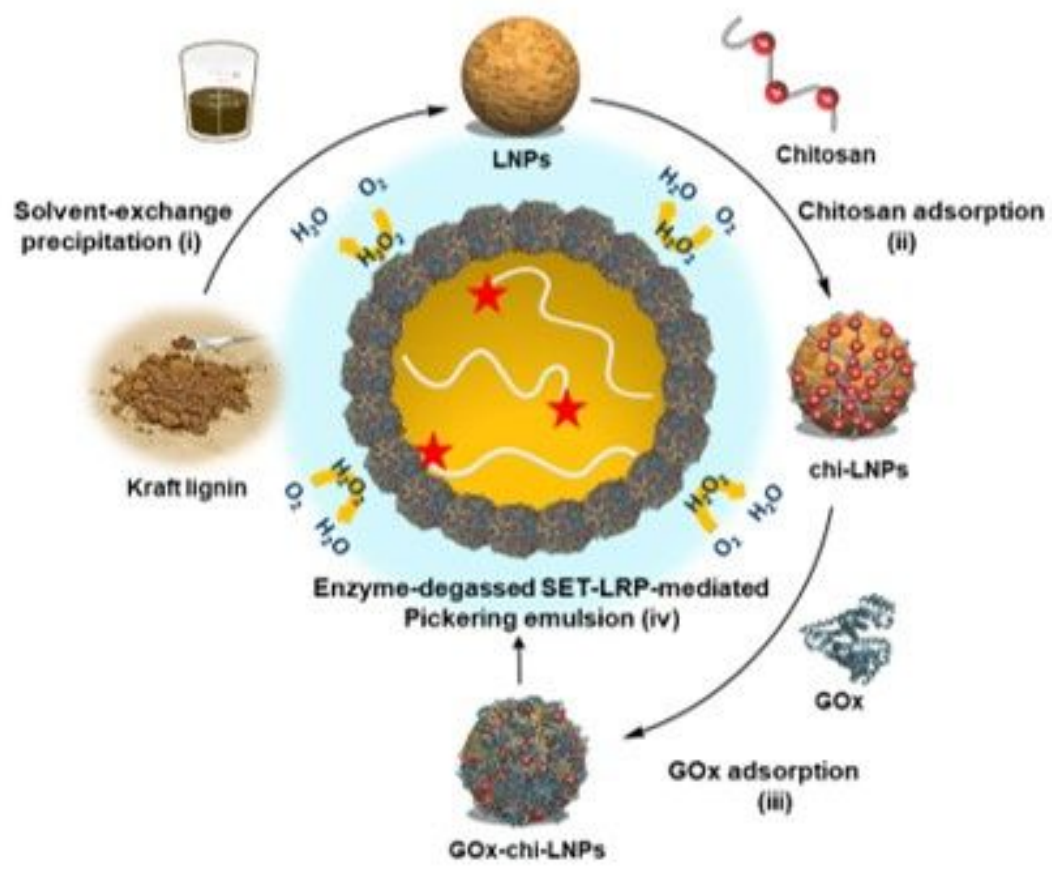

\section{Figure 1}

General process scheme for the preparation of GOx-chi-LNPs via two-step adsorption immobilization and application in enzyme-degassed SET-LRP -mediated Pickering emulsion process: (i) Preparation of LNPs via solvent-exchange precipitation. (ii) adsorption of chitosan on LNPs to yield chi-LNPs. (iii) Adsorption of GOx on chi-LNPs to yield GOx-chi-LNPs. (iv) Application of GOx-chi-LNPs as enzyme-degassed stabilizers for SET-LRP in Pickering emulsion media.

a

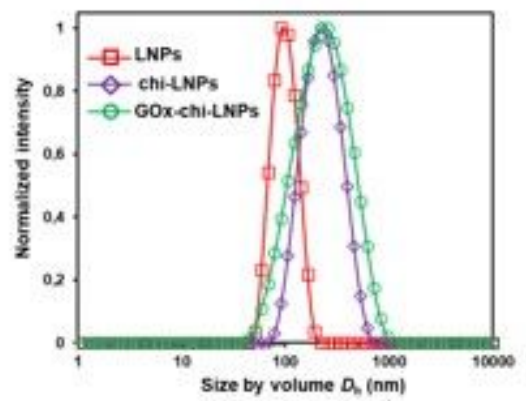

C

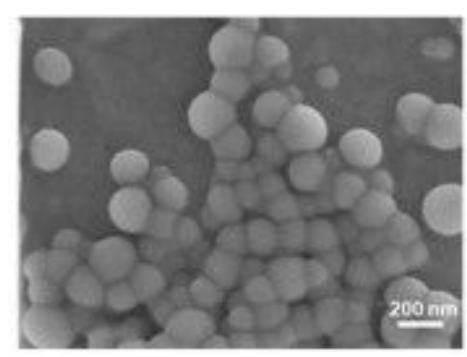

b

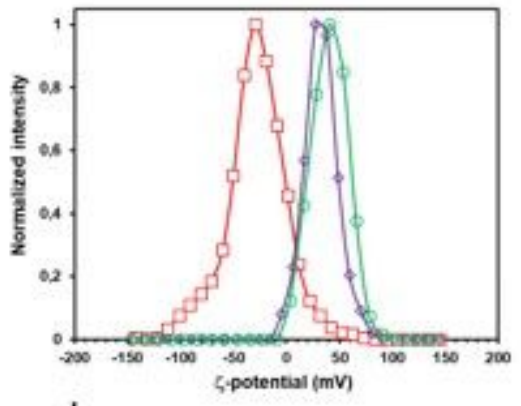

d

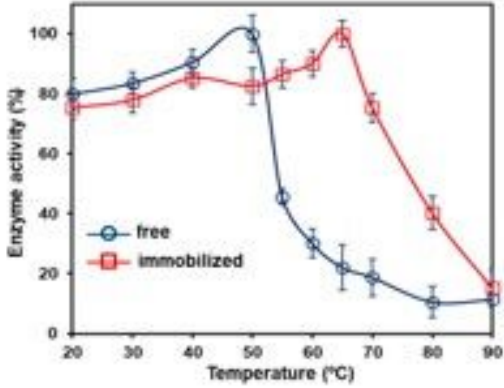

Figure 2 
(a) Hydrodynamic diameter and (b) Z-potential distributions of lignin nanoparticles employed in this work. $(\square)$ LNPs. $(\mathbb{\nabla})$ chi-CLPs and ( $)$ GOx-chi-CLPs. (c) SEM image of GOx-chi-LNPs. (d) Thermal stability of $(\bullet)$ free and ( $\square)$ immobilized GOx.
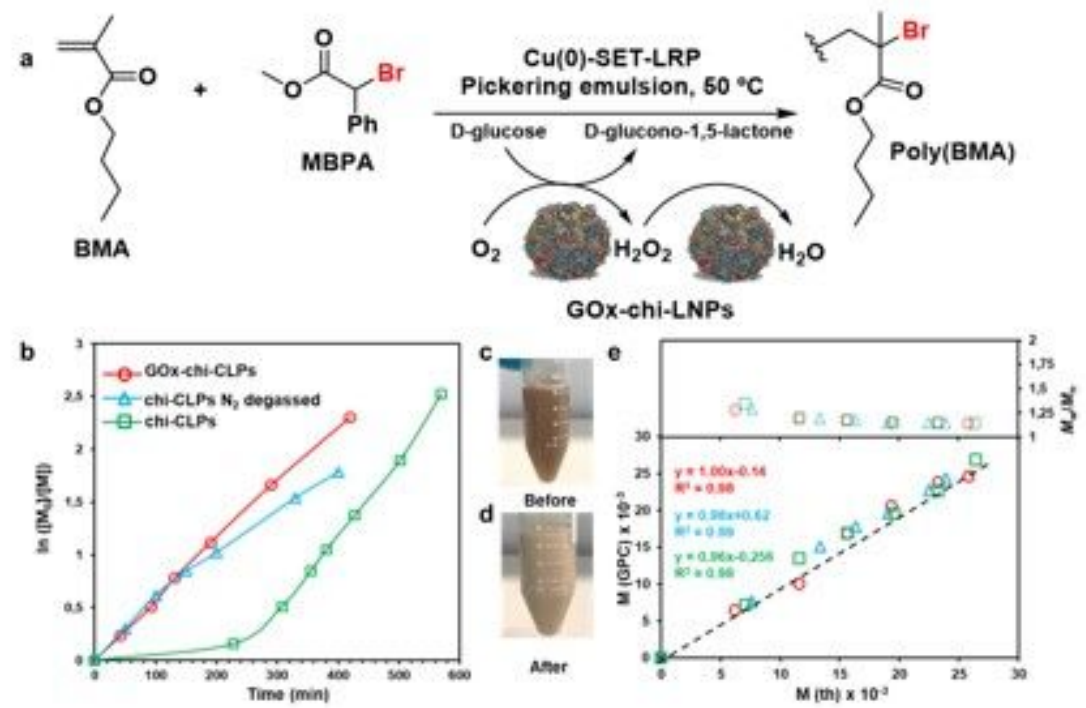

\section{Figure 3}

Evaluation of the enzyme-degassed controlled polymerization using GOx-chi-LNPs as stabilizers: (a) Scheme of enzymatic-degassed SET-LRP -mediated Pickering emulsion of BMA. (b and e) Kinetic plots and evolution of experimental Mn (GPC) and Mn/Mw vs theoretical Mnth for the SET-LRP-mediated Pickering emulsion of BMA using chi-CLPs $(\square)$, GOx-chi-CLPs $(\bullet)$ in the presence of oxygen, and chiCLPs $(\triangle)$ in N2 degassed emulsion. (c and d) Digital images of BMA-based Pickering emulsions before (c) and after (d) SET-LRP process using GOx-chi-CLPs as stabilizers. Reaction conditions:

$[\mathrm{BMA}] 0 /[\mathrm{MBPA}] 0 /[\mathrm{Me} 6-\mathrm{TREN}] 0 /[\mathrm{Cu}(0)] 0=200 / 1 / 0.2 / 0.3$. [Lignin nanoparticles] $=2.25 \mathrm{wt} \%$ relative to BMA.
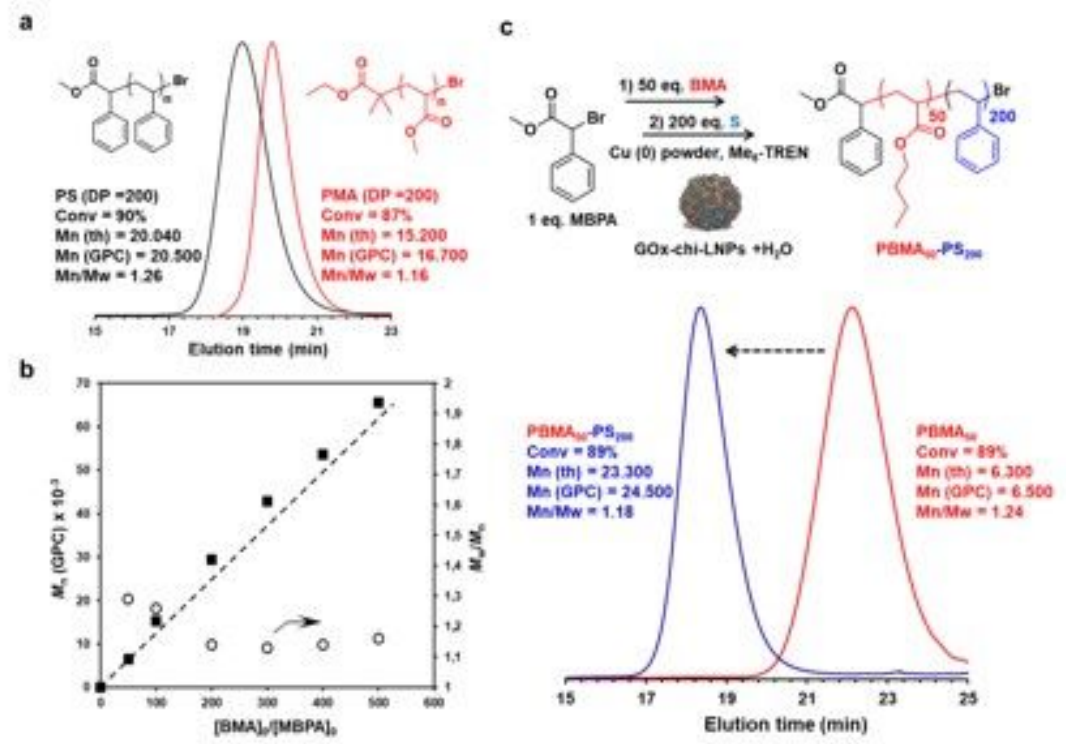


\section{Figure 4}

(a) GPC curves of PMA and PS obtained by enzyme-degassed Pickering emulsion SET-LRP. Reaction conditions: $[$ Monomer]0/[MBPA or EBiB]0/[Me6-TREN]0/[Cu(0)]0 = 200/1/0.2/0.3. [Lignin nanoparticles] $=$ $2.25 \mathrm{wt} \%$ relative to MA or S. (b) Dependence of experimental Mn (GPC) and Mw/Mn on the [BMA]0/[MBPA]0 ratio. (c) GPC analysis of block copolymerization via purification-reinitiation strategy of PBMA with styrene (S) to synthetize PBMA-PS block copolymer. Reaction conditions for the PBMA macroinitiator: $[\mathrm{BMA}] 0 /[\mathrm{MBPA}] 0 /[\mathrm{Me} 6-\mathrm{TREN}] 0 /[\mathrm{Cu}(0)] 0=50 / 1 / 0.2 / 0.3 .[$ GOx-chi-LNPs] $=2.25$ wt \% relative to BMA.

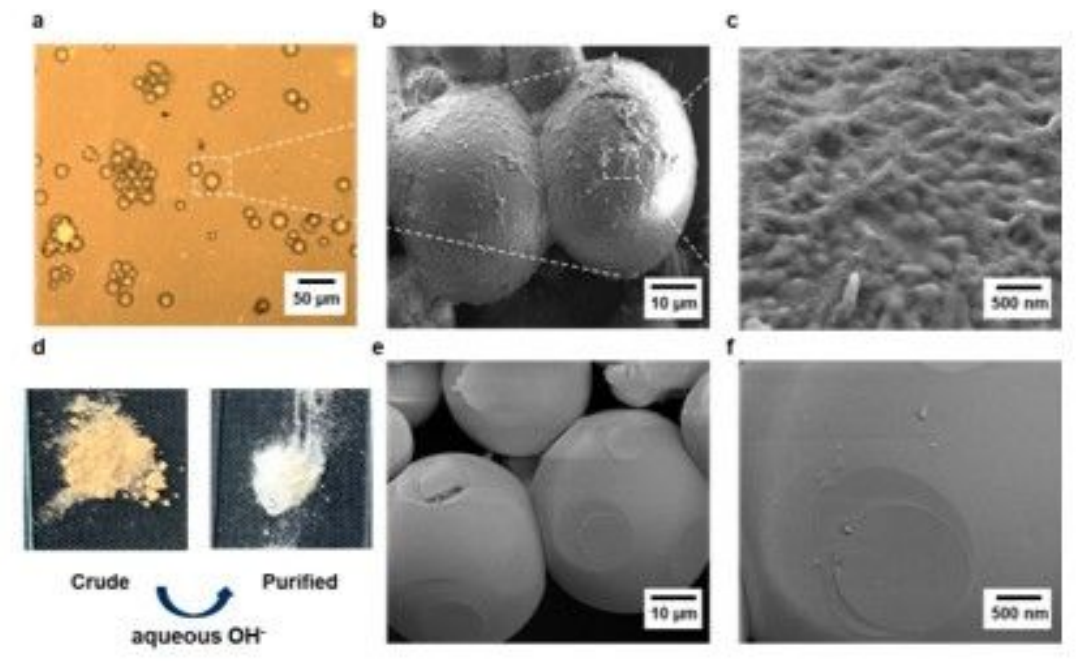

\section{Figure 5}

(a) GPC curves of PMA and PS obtained by enzyme-degassed Pickering emulsion SET-LRP. Reaction conditions: [Monomer]0/[MBPA or EBiB]0/[Me6-TREN]0/[Cu(0)]0 $=200 / 1 / 0.2 / 0.3$. [Lignin nanoparticles] $=$ $2.25 \mathrm{wt} \%$ relative to MA or S. (b) Dependence of experimental Mn (GPC) and Mw/Mn on the [BMA]0/[MBPA]0 ratio. (c) GPC analysis of block copolymerization via purification-reinitiation strategy of PBMA with styrene (S) to synthetize PBMA-PS block copolymer. Reaction conditions for the PBMA macroinitiator: $[\mathrm{BMA}] 0 /[\mathrm{MBPA}] 0 /[\mathrm{Me} 6-\mathrm{TREN}] 0 /[\mathrm{Cu}(0)] 0=50 / 1 / 0.2 / 0.3$. [GOx-chi-LNPs] $=2.25 \mathrm{wt} \%$ relative to BMA.

\section{Supplementary Files}

This is a list of supplementary files associated with this preprint. Click to download.

- Supportinglnformation.pdf 